\title{
LA INTERACCIÓN DOCENTE-DISCENTE EN CONTEXTOS ESCOLARES CON FUERTE PRESENCIA DE ALUMNOS DE FAMILIA INMIGRANTE: PROPUESTAS DE LA INVESTIGACIÓN
}

\author{
Joaquín Gairín Sallán * \\ Edgar Iglesias Vidal **
}

SÍNTESIS: La presente aportación presenta los resultados más relevantes de una investigación ${ }^{1}$ realizada, entre los meses de marzo de 2006 y de abril de 2007, por un equipo ${ }^{2}$ integrado por profesores universitarios del Departamento de Pedagogía Aplicada de la UAB y profesores no universitarios, focalizada en el análisis de modelos de interacción en contextos con fuerte presencia de inmigrantes de reciente incorporación al sistema educativo.

Se presentan, en primer lugar, algunos de los aspectos del diseño y metodología utilizados con el fin de situar y contextualizar, en la segunda parte del texto, una selección de las propuestas más relevantes que se realizan y que están referidas a los procesos de enseñanza-aprendizaje, organización del aula, agentes educativos, contexto del centro y sistema educativo; también se enfatiza en el aspecto dinámico de los procesos de integración y en la necesidad de atender aspectos de naturaleza emocional.

* Catedrático de Didáctica y Organización Escolar en la Universidad Autónoma de Barcelona (UAB) y decano de la Facultad de Ciencias de la Educación de la misma casa de altos estudios.

** Miembro investigador de dos equipos: del ERIC (Equip de Recerca en Educació Intercultural) adscripto al Instituto de Ciencias de la Educación (ICE) de la UAB y del GREI (Grup de Recerca en Educació Intercultural), adscrito al Equipo de Desarrollo Organizativo (EDO) del Departamento de Pedagogía Aplicada de la mencionada universidad.

${ }^{1}$ La investigación «La interacción docente-discente en contextos escolares con fuerte presencia de alumnado de familia inmigrante» corresponde a un proyecto competitivo ARIE (Investigación e innovación en materia educativa y de enseñanza formal [reglada] y no formal [no reglada]), convocado por Resolución UNI/2414/2005, de 29 de julio (Diario Oficial de la Generalitat de Catalunya [DOGC] n. ${ }^{\circ} 4.456$, de 26 de agosto de 2005) y subvencionado según Resolución del 6 de marzo de 2006.

${ }^{2}$ El equipo de investigación estuvo formado (en orden alfabético) por Gemma Carreras, Esther Collados, Miquel A. Essomba, Joaquín Gairín (coordinador), Edgar Iglesias, Cristina Laborda, Anna Oliveras, Pere Peris, Cynthia Sabaté, Susana Tovías y Gloria Valls. 
El objetivo final es, pues, el de ofrecer propuestas de mejora desde la perspectiva de la educación intercultural que favorezcan el proceso de integración con la inclusión plena al sistema educativo de los estudiantes de origen inmigrante.

Palabras clave: procesos de interacción docente-discente; estudiantes de origen inmigrante; enseñanza-aprendizaje de inmigrantes.

SINTESE: A presente proposta consiste em apresentar os resultados mais relevantes de uma pesquisa ${ }^{3}$ realizada, entre os meses de março de 2006 e abril de 2007, por uma equipe 4 integrada por professores universitários do Departamento de Pedagogia Aplicada da Universidade Autônoma de Barcelona (UAB) e por não universitários, centrada na análise de modelos de interação em contextos com forte presença de imigrantes de recente incorporação ao sistema educativo.

Apresentam-se, em primeiro lugar, alguns dos aspectos do projeto e da metodologia utilizados a fim de situar e contextualizar a pesquisa, e na segunda parte do texto, uma seleção das propostas mais relevantes que se realizam e que se referem aos processos de ensino-aprendizagem, organização da aula, agentes educativos, contexto do centro e sistema educativo; também se enfatizam o aspecto dinâmico dos processos de integração e a necessidade de atender questões de natureza emocional O objetivo final é, portanto, oferecer propostas de melhoria a partir da perspectiva da educação intercultural que favoreça o processo de integração com a inclusão plena no sistema educativo dos estudantes de origem imigrante.

Palavras-chave: processos de interação docente-discente; estudantes de origem imigrante; ensino-aprendizagem de imigrantes.

ABSTRACT: The present contribution presents the most relevant results of a research ${ }^{5}$ carried out between March 2006 and April 2007,

${ }^{3} A$ pesquisa «A interação docente-discente em contextos escolares com forte presença de alunos de família imigrante» corresponde a um projeto competitivo ARIE (Investigação e inovação em matéria educativa e de ensino formal [regulado] e não formal [não regulado]), convocado pela Resolução UNI/2414/2005, de 29 de julho, (Diário Oficial da Generalidade de Catalunha (DOGC) n. ${ }^{\circ} 4.456$, de 26 de agosto de 2005) e subvencionado conforme a Resolução de 6 de março de 2006.

${ }^{4}$ A equipe de pesquisa esteve formada (em ordem alfabética) por Gemma Carreras, Esther Colados, Miquel A. Essomba, Joaquin Gairín (coordenador), Edgar Iglesias, Cristina Laborda, Anna Oliveras, Pere Peris, Cynthia Sabaté, Susana Tovías e Gloria Valls.

${ }^{5}$ The research «Teacher-student interaction in school contexts with strong presence of students from immigrating families» belongs to a competitive ARIE project (Research and innovation in education, and formal and informal education) summoned by Resolution UNI/2414/2005 from July $29^{\text {th }}$ (Official Bulletin of Catalonia [DOGC for its Spanish acronym] number 4.456 from August $26^{\text {th }} 2005$ ) and funded according to the Resolution from March $6^{\text {th }} 2006$. 
by a team ${ }^{6}$ of college professors from the Department of Applied Pedagogy of the Autonomous University of Barcelona (UAB for its Spanish acronym) and by teachers of other levels. The research is focused on the analysis of integration models in contexts that have a strong presence of immigrants that have recently been incorporated into the educative system.

We will firstly present a few aspects concerned with the design and the method used, with the goal of giving a background to the choice of the most relevant proposals presented in the second part of the paper. These proposals refer to teaching-learning processes, classroom organization, educative agents, background of center and the educative system. We will also underline the dynamics of the integration processes and the need for dealing with aspects of an emotional nature.

The final goal is, therefore, to offer proposals for improvement, from the point of view of intercultural education. These proposals should facilitate the integration process by fully including students of immigrating families into the educative system.

Keywords: teacher-student interaction processes; students from immigrating families; teaching-learning for immigrants.

\section{ANTECEDENTES Y FUNDAMENTACIÓN TEÓRICA}

Los flujos migratorios son motivados, mayoritariamente, por razones económicas y se traducen en la concreción de proyectos migratorios familiares. Suponen, y continuarán suponiendo, un incremento y presencia notables de hijos de familias de origen inmigrante en las aulas que configuran y forman parte del sistema educativo español. Esta realidad se ha presentado juntamente con, por un lado, algunas deficiencias estructurales ya existentes en el sistema educativo, y por otro, el alarmante fracaso escolar en el marco de la educación secundaria. Nos situamos en una de las cuestiones educativas prioritarias y preocupantes en el espacio de la educación, que tratan de resolver tanto el conjunto de la comunidad educativa como la comunidad científica y académica.

Sin embargo, y en concreto en Cataluña, esta preocupación no siempre se ha visto acompañada de planteamientos de acción suficientemente sólidos y rigurosos, sino que, más bien, en los últimos años se

6 The research team was constituted by the following professionals (in alphabetical order) Gemma Carreras, Esther Collados, Miquel A. Essomba, Joaquín Gairín (coordinator), Edgar Iglesias, Cristina Laborda, Anna Oliveras, Pere Peris, Cynthia Sabaté, Susana Tovías and Gloria Valls. 
evidenció un cierto desconcierto sobre cómo abordar esta cuestión. EI sistema educativo catalán, tradicionalmente selectivo y propedéutico como el del resto de las comunidades autónomas del Estado español, mantiene el reto de extender la escolarización obligatoria, ofreciendo una educación de calidad para toda la población hasta los 16 años. Este propósito se desarrolla en el marco de un escenario poco favorable donde, actuando como barreras o escollos que dificultan su consecución, se hacen presentes la existencia de un currículum y de una cultura marcadamente etnocéntricos, junto a un cuerpo docente con déficits formativos en relación a los contenidos interculturales 0 un exceso de burocratización.

Las políticas educativas en Cataluña han evidenciado, en estos últimos años, desorientación y presión en esta temática. Si en un primer momento las autoridades educativas optaron por políticas claramente asimilacionistas, justificadas a partir de una supuesta igualdad -todo estudiante de origen inmigrante debía recibir el mismo trato que sus compañeros para evitar el riesgo de ser discriminado a partir de la diferenciación- posteriormente, y ante la oposición de muchos docentes a este modelo, se optó por planteamientos segregadores a partir del establecimiento de aulas especializadas y de programas específicos, separados de los habituales y propios de los centros ordinarios. Finalmente, se renunció a este último planteamiento al considerar que la inclusión posterior de los estudiantes inmigrantes al aula ordinaria resultaba un fracaso, justificando así la instauración de las actuales políticas educativas, de carácter más integrador e inclusivo.

Se ha comprendido que no es adecuado ni justo marginar y segregar a estos estudiantes de los centros ordinarios y que las medidas educativas necesarias no han de ser homogéneas, sino que han de ofrecer respuestas a las necesidades educativas de cada uno de ellos. Actualmente, las autoridades educativas promueven para los estudiantes la existencia de planes de acogida, de sistemas diversificados de atención a las diferentes necesidades lingüísticas, comunicativas y curriculares -no siempre coincidentes-y establecen materiales de soporte, así como la incorporación de especialistas bajo la figura de mediadores culturales o psicopedagogos, que facilitan el proceso integrador. En resumen, se avanza lentamente en el camino de la inclusión al mismo tiempo que se reconoce que el mismo no está exento de dificultades y retos.

Algunos de los estudios realizados desde diferentes ámbitos como la sociología, antropología o pedagogía, ratifican nuestras anterio- 
res apreciaciones. Así, y en clave de ejemplo, podemos destacar los estudios de Lorenzo (1999), Carbonell (2000), Sipán (2001), Giménez y Malgesini (2002), García (2002), Fullana, Besalú y Vilà (2003), Comas (2003), Conteh (2003), Suárez-Orozco y Suárez-Orozco (2003), Garreta (2003), Montón (2003), Fuster (2004), Bonal y otros (2004), Besalú y Climent (2004), Jordán (2004), Pujolàs (2004), EURYDICE (2005), Carbonell, Simó y Tort (2005), Torrabadella y Tejero (2005).

\section{CONTEXTUALIZACIÓN}

La investigación configura sus objetivos, metodología, estudio de campo y análisis de la información desde una dimensión práctica y aplicable. En el marco de la interacción entre docente y discente en centros educativos de primaria y secundaria, se han tratado de detectar y señalar algunos de los elementos incipientes que se relacionan tanto en la práctica y función docente como en la actitud y percepción de los estudiantes, que inciden en el marco de la misma interacción. Se trata de comprender a esta desde su dimensión dinámica e interactiva, considerando la dimensión procesual como el espacio donde sus protagonistas se configuran como agentes culturales activos, con un bagaje y experiencia personales amplios y diversos. Así, el proceso de interacción se fundamenta y analiza en una doble dimensión: por un lado, se consideran las percepciones de unos y otros agentes, $y$, por otro y en función de las mismas, se analizan las distintas formas de interacción, de tal forma que las actitudes, percepciones y comportamientos van situándose de acuerdo a la naturaleza del proceso interactivo.

En este marco, y con el fin de establecer y ofrecer posteriormente propuestas de mejora, el grupo de investigación recoge algunos elementos explicativos sobre la distancia existente entre el discurso y la práctica. Aunque se considera que el objeto de estudio se sitúa en el marco de los procesos de interacción, en relación a la existencia de elementos condicionantes en la producción, mantenimiento y proyección de determinados modelos de interacción, se asume también la premisa de que estos procesos son condicionados y alimentados a partir de elementos de naturaleza interna y externa y en relación al espacio físico donde se produce la misma interacción.

De este modo, se justifica el que sean objeto de análisis y observación los elementos que permiten identificarse en el proceso de la 
interacción y que tienen su origen en el proceso de enseñanza-aprendizaje, estableciendo posibles relaciones entre los mismos modelos y los resultados formativos de los estudiantes. Asimismo, se relacionan elementos fundamentados a partir de la estructura, capacidad organizativa y normativa del centro, de la estructura del sistema educativo y de la forma de disponer el aula, por considerarlos relacionados con el marco de los procesos de interacción. Consideramos que las relaciones establecidas a partir del análisis del discurso, tanto de los actores implicados como de los agentes del sistema educativo, son una aproximación válida para analizar las atribuciones de significado que se dan en este proceso interactivo.

\section{DISEÑO Y DESARROLLO DEL ESTUDIO}

Los objetivos principales de la investigación son:

- Detectar los elementos incipientes y característicos de diferente naturaleza que inciden en el marco de la interacción entre docentes y discentes de origen inmigrante.

- Analizar los efectos de las interacciones en docentes y discentes.

- Elaborar propuestas de mejora desde la perspectiva de la educación intercultural.

Algunas concreciones iniciales a considerar nos permiten situar el objeto de estudio y su naturaleza:

- La categoría de «alumnos inmigrantes» se aplica a estudiantes de origen extranjero con una permanencia menor de tres años en el contexto socio-educativo que se analiza.

- Las expectativas mutuas generadas entre docentes y estudiantes se tienen en cuenta en el marco de la interacción, así como también las expectativas del contexto familiar de los estudiantes hacia el nuevo contexto educativo.

- El grupo de investigación se configuró considerando la necesidad e importancia de la proximidad al objeto de estudio. Así, cinco de sus miembros son docentes e investi- 
gadores del Departamento de Pedagogía Aplicada y seis son docentes de distintos centros de primaria y secundaria.

El planteamiento metodológico combina una aproximación general (a partir de cuestionarios) y una aproximación específica. En todos los casos se consideran cinco unidades de análisis teniendo en cuenta la interrelación de los elementos presentes: contexto aula, contexto enseñanza-aprendizaje, contexto agentes, contexto centro y contexto sistema. Cada uno de los contextos mencionados se analiza a partir de variables comunes y específicas (véase en el Anexo: documento matriz, páginas 248-250), que han ofrecido distintos espacios para orientar la recogida de los datos y la posterior discusión y elaboración de propuestas.

Las fases generales de la investigación han sido:

- Revisión de la literatura científica. Realizada a partir de las variables que configuran las unidades de análisis del documento matriz.

- Trabajo de campo. Consistente en la elaboración y aplicación de los instrumentos para la recogida de la información.

- Delimitación de resultados. Incluye sintetizar y tratar la información con procedimientos analíticos y sincréticos.

- Elaboración de conclusiones y discusión. Realizada mediante la:

- Identificación de las convergencias y de las divergencias entre las informaciones recogidas durante el trabajo de campo.

- Contrastación entre los datos e informaciones recogidas durante el trabajo de campo y las aportaciones teóricas.

- Valoración. Interpretación y posicionamiento delante de los resultados obtenidos y debatidos.

- Elaboración de propuestas específicas para cada unidad de análisis, en función de las valoraciones realizadas.

La muestra definitiva que configura el estudio es de un total de 36 centros educativos de educación primaria y secundaria de diferentes municipios y/o ciudades de Cataluña. Para la selección se aplicó el 
criterio de que, como mínimo, el $20 \%$ de estudiantes del centro educativo fuera de origen inmigrante y los municipios y/o ciudades de referencia contaran como mínimo con el $15 \%$ de ciudadanos inmigrantes en relación a su población total.

El estudio de campo ha exigido la aplicación de variados instrumentos para la recogida de información, referenciados en la matriz de información recogida en el Anexo. Más concretamente, podemos referenciar como fuentes de información:

261 cuestionarios de docentes de centros educativos de primaria y secundaria, situados en municipios de la provincia de Barcelona.

27 observaciones de aula que incluyen cada una entre tres y cuatro momentos de estancia en ella y con el mismo tutor.

10 grupos de discusión en los centros educativos donde se realizó el trabajo de campo.

22 entrevistas en profundidad dirigidas a los docentes de las aulas donde se realizaron las observaciones.

56 historias de vida de estudiantes relacionadas con aspectos vivenciales de la escolarización e interacción con docentes y compañeros.

18 entrevistas en profundidad dirigidas a personas expertas del sistema educativo: docentes universitarios especializados en diversidad cultural, expertos en inmigración y responsables técnicos y/o políticos del sistema educativo.

Las historias vivenciales y las observaciones de aula permiten acercarse directamente a la categoría discente. Asimismo, los cuestionarios y las entrevistas lo hacen en relación al docente, mientras que los grupos de discusión y las entrevistas a expertos permiten validar y/o contrastar algunas de las informaciones recogidas, evidenciadas y manifestadas por parte de discentes y docentes.

\section{RESULTADOS Y PROPUESTAS PARA LA MEJORA}

A continuación se presentan resumidas algunas de las propuestas específicas de acuerdo a las unidades de análisis consideradas. 


\subsection{EN RELACIÓN AL CONTEXTO AULA}

Resulta necesario y conveniente acompañar a los estudiantes recién incorporados y que se encuentran desvinculados y poco identificados con el aula, si queremos lograr procesos de inclusión efectivos.

Esta necesidad de acompañamiento se justifica desde varias perspectivas. En primer lugar, a lo largo de la investigación se constata y evidencia que el primer período escolar del estudiante de origen inmigrante puede resultar complejo y que una de las razones de esta complejidad es de naturaleza emocional. Se da una falta de ubicación frente al nuevo escenario social, cultural y académico, así como manifestaciones de desvinculación hacia el aula o dificultades de comprensión relativas al sentido de las tareas asignadas.

Igualmente, se constata un desequilibrio entre algunas vivencias y consideraciones sobre este primer momento en la escuela. Mientras que los estudiantes manifiestan que se trata de una instancia especialmente dura, los responsables y expertos del sistema sostienen que esta problemática se sitúa más bien en etapas posteriores.

Los docentes muestran una preocupación vinculada a la falta de información previa sobre el contexto social, económico y académico del estudiante de reciente incorporación. Cubrir esta necesidad permitiría situar mejor al docente con el fin de comprender más profundamente la experiencia personal del estudiante. Esta necesidad se evidencia más en la educación secundaria, donde los cambios y movilidad de los estudiantes son mayores y se acompañan de variabilidad de situaciones y de dificultades para conocer dicha información.

Los estudiantes manifiestan vivencias negativas originadas a partir de expresiones de discriminación, y en algunos casos, los profesores no son capaces de percibirlas, tal y como se recoge a partir de la siguiente afirmación relativa a una historia de vida: «Sí, tengo la sensación de estar discriminada, pero trato de olvidar» ([3HV5] mujer, 15 años, país de origen: Bolivia).

Una explicación para algunas de las situaciones planteadas se puede relacionar con la interpretación que hacen algunos profesores respecto a la asignación de algunos conflictos como responsabilidad única del estudiante, sin que sean vividos, experimentados o valorados de esta forma por los mismos estudiantes. En estos casos se recomienda 
que el posible conflicto sea entendido como un hecho normal y como una oportunidad para asumir la responsabilidad compartida, aconsejando que cuando el mismo se manifieste sea abordado colectivamente.

Por otro lado, los procesos de interacción observados han permitido identificar elementos de diferenciación en función del origen de los inmigrantes. Este hecho no obvia la necesidad de establecer procesos profundos de reconocimiento, trabajando, por ejemplo, el vínculo de pertenencia del estudiante al centro o abordando detenidamente lo que el mismo expresa; también compartiendo aquellos aspectos relativos a su origen que los estudiantes manifiestan de manera mayoritaria que desearían socializar. De esta forma, se tienen en cuenta ciertos aspectos que intervienen en la realidad del alumno, como relaciones familiares o la cohesión de grupos según la variable cultural o étnica.

Reiteramos, por tanto, que la actitud del docente es un elemento básico y prioritario, pues refleja y es portadora de modelos de relación, influyendo en la percepción del alumno y en la construcción de los vínculos emocionales que se desarrollan. Es indispensable, en este sentido, que el docente se muestre activo y se ofrezca como herramienta de apoyo al estudiante, ya que es durante los primeros días y los posteriores al proceso de incorporación del estudiante cuando se pone de relieve la existencia de un importante interés por parte del grupo de compañeros respecto al recién llegado, que disminuye con el tiempo.

Recomendamos, así, que la acogida y acompañamiento se realicen trabajando con y para el grupo. La tarea no puede ser únicamente responsabilidad del docente pues son los vínculos emocionales, importante motor de las interacciones, los que permitirán y favorecerán este proceso de interacción e inclusión. La potenciación de grupos heterogéneos, tanto en el aula como en los espacios informales del centro, es una iniciativa positiva, que permite que la regulación que realiza el docente en el marco de la interacción se enriquezca y compense la situación externa al ámbito escolar, donde las interacciones y vínculos entre alumnos de distintos orígenes disminuyen.

\subsection{EN RELACIÓN AL CONTEXTO ENSEÑANZA-APRENDIZAJE}

Desde una perspectiva inclusiva resulta necesario dinamizar la creación, difusión y utilización adecuada de materiales didácticos de referencia y relacionados con la actividad en el aula. 
La investigación constata que gran parte de los materiales curriculares y didácticos utilizados en las aulas son uniformes y estandarizados. Se evidencia en ellos un importante etnocentrismo, así como la proyección de visiones monoculturales que reflejan la construcción y permanencia de la categoría mayoría-minoría. En general, los estudiantes utilizan los mismos textos pensados para el alumno autóctono, que en muchos casos no responden a las necesidades del estudiante inmigrante, al reproducir tópicos y visiones etnocéntricas. A modo de ejemplo, se recoge la opinión de un experto del sistema educativo entrevistado: «Los libros de textos de asignaturas ordinarias no consideran las necesidades del alumnado inmigrante pues reproducen tópicos etnocéntricos» ([ERS2] experto en la temática).

La situación general descrita no soslaya la existencia de materiales específicos sobre algunos países, como mapas o libros de lectura. Un comentario tomado de una historia de vida es ilustrativo al respecto: «Aparte de los mapas no hay materiales propios de mi cultura, aunque los profesores saben que vengo del otro lado del mundo y, por lo tanto, no voy a ser igual que ellos» ([3HV38] varón, 14 años, país de origen: Bolivia).

Esta falta de referencias al contexto de origen o a la realidad personal de los estudiantes de reciente incorporación al sistema escolar se da también en los objetivos y en los contenidos curriculares, que frecuentemente no son elaborados por docentes y sí proporcionados por las editoriales. El hecho de seguir estrictamente los libros de texto obvia la consideración de los objetivos mínimos establecidos oficialmente y no invita ni a establecer diferencias ni a compensar desigualdades. Más en concreto y en la educación secundaria, donde cada profesor atiende a un alto número de estudiantes, se priorizan los materiales estandarizados y estos condicionan los objetivos reales que acompañan el proceso de enseñanza-aprendizaje. Se recomienda, en estas circunstancias y ante la ausencia de materiales adaptados, que el docente utilice más de un material, para que pueda hacer efectiva la posibilidad de reorientar algunos de sus contenidos.

La tendencia a abordar cuestiones del país de origen, por el contrario, sí se da en el aula de acogida, constatando que esta realidad está establecida y formalizada. Puntualmente, puede darse en el aula ordinaria aunque no se acostumbra a programar dichas cuestiones con anterioridad. Se constata que, de manera generalizada, los estudiantes valoran y recuerdan positivamente el hecho puntual de que en el aula 
ordinaria se traten cuestiones relacionadas con su origen o bagaje cultural.

Se identifican, pues, necesidades formativas de los docentes, tanto respecto a la orientación y uso de los materiales como en relación al desarrollo y atribución de significado de los fundamentos de la educación intercultural. Docentes y expertos del sistema reconocen de manera generalizada esta necesidad formativa, al mismo tiempo que evidencian la existencia actual de una formación puntual y de iniciativa personal por parte de los profesores más sensibilizados; asimismo, algunos docentes manifiestan que la formación se ha orientado desde una dimensión didáctica y poco ideológica, asumiendo que se debería poner mayor atención en cuestiones como la actitud y el compromiso.

En resumen, se recomienda que todos los materiales se confeccionen y utilicen a partir de la perspectiva y principios de la educación intercultural y que favorezcan la interacción entre el grupo de estudiantes. También se apuntan como propuestas complementarias la promoción de proyectos de investigación sobre los materiales didácticos según el modelo de investigación-acción, la incentivación de asesoramientos relativos a la utilización de los materiales y la potenciación del ámbito intercultural en los centros de recursos.

\subsection{EN RELACIÓN AL CONTEXTO CENTRO}

Resulta necesaria la revisión de algunos aspectos del aula de acogida, con la finalidad de que sea un espacio abierto, flexible e inclusivo.

El aula de acogida ha aparecido, a lo largo de la investigación, como un espacio y, a la vez, como una medida educativa presente tanto para los docentes como para los estudiantes participantes en el estudio. Su utilización, donde existe, es frecuente y se menciona de una manera constante y reiterativa. Se constata que en ella se genera y facilita un clima agradable de respeto y trabajo, posibilitado por una distribución de espacios según unidades didácticas o centros de interés -por ejemplo, lectura o trabajo con herramientas multimedia-, y por contar con un grupo reducido de estudiantes.

Las aulas de acogida son valoradas de una forma positiva por los estudiantes de reciente incorporación; asimismo, manifiestan que el 
trabajar con grupos reducidos potencia la interacción entre estudiantes y permite realizar referencias constantes a elementos culturales relacionados con su procedencia geográfica y cultural. En estas aulas, además, suelen encontrarse y utilizarse materiales específicos, orientados a distintos niveles y clasificados por apartados y por objetivos de aprendizaje.

Se constata la necesidad de que el profesional del aula de acogida tenga un peso específico dentro el claustro de profesores, con la finalidad de facilitar el establecimiento de sinergias y el trabajo conjunto, evitando así que su acción e influencia quede limitada solo al espacio físico de la misma. Esta integración permanente y real al claustro se justifica por su capacidad para recoger información relevante acerca de los estudiantes, relacionada con aspectos de naturaleza emocional y de su proceso de enseñanza-aprendizaje.

También se constata, en algunas ocasiones, la necesidad de una mayor coordinación y comunicación entre el profesional del aula de acogida y los respectivos tutores de los estudiantes. A veces se dificulta esta coordinación sistemática y permanente por la propia dinámica del centro educativo, que limita la posibilidad de mantener espacios específicos para la reflexión sobre la función docente.

Se recomienda que el aula de acogida sea concebida como un recurso más y no como parte de un proceso de adaptación gradual del estudiante, donde la prioridad única no sea su escolarización. Debe, además, evitar ser segregadora y conseguir dinámicas positivas en constante interacción con el centro educativo. Otra medida complementaria a la mencionada es la posibilidad de elaborar proyectos comunes entre los estudiantes, promoviendo la interacción entre distintos grupos y evitando centrarse casi exclusivamente en aspectos del aprendizaje del idioma.

\subsection{EN RELACIÓN AL CONTEXTO SISTEMA}

Resulta necesario potenciar y promover aquellas actuaciones que permitan y garanticen la interacción entre centro y entorno: tejido social-familias-centro-alumnado.

La investigación muestra la tendencia de los centros educativos a abrirse gradualmente al entorno aprovechando cada vez más tanto los 
recursos internos del centro como los externos del entorno. Son conscientes de la idoneidad de la apertura y muestran su satisfacción en relación a las experiencias innovadoras que se dirigen a establecer sinergias y trabajo conjunto entre el centro educativo y su entorno.

Sin embargo, los procesos de apertura resultan complejos y algunos elementos propios de las dinámicas institucionales de los centros educativos, como la presión y el alto ritmo de trabajo, pueden generar dificultades. Ante ello, cabe mejorar y potenciar la coordinación y comunicación con algunos de los agentes externos implicados en la inclusión socio-cultural de los estudiantes de reciente incorporación, tratando de este modo que el centro educativo sea también una parte activa en su proceso de socialización.

Resulta así recomendable abordar la utilización de los espacios informales. Es interesante que se promuevan aquellas acciones docentes que permitan y garanticen la interacción y relación entre estudiantes tanto dentro como fuera del centro educativo. Particularmente y en la educación secundaria, este propósito se convierte en esencial, pues los vínculos de los estudiantes dentro del centro pueden ser únicamente académicos, y promover la interacción fuera el ámbito escolar puede resultar beneficioso para su integración social y emocional. Algunos expertos del sistema educativo proponen, en este sentido, que se prioricen las subvenciones a los centros educativos que ofrezcan actividades extraescolares que reflejen la diversidad del alumnado.

El bajo grado de relación entre el centro y la familia se relaciona con causas como el desconocimiento del sistema educativo, las expectativas escolares diferentes, la dificultad para compatibilizar horarios, el desconocimiento cultural compartido o la priorización de la comunicación escrita. No obstante, los docentes proporcionan mayoritariamente una valoración positiva acerca del esfuerzo de las familias por implicarse y comunicarse con el centro.

Mejorar la comunicación exige detectar y comprender mejor cuáles son las expectativas de las familias, sin obviar la reflexión, a partir de un cierto ejercicio de introspección, sobre las del centro y las de los docentes, pues todas las expectativas son importantes y deben contemplarse en el marco de la interacción. Conviene que el centro educativo adecúe la tipología del lenguaje a la familia, sin utilizar tecnicismos innecesarios o partiendo de la premisa falsa de que las familias no entenderán el idioma; en este sentido, una medida complementaria e 
interesante es incorporar la figura del traductor. Asimismo, existe la necesidad de mejorar el tipo de acogida a las familias por parte del centro, distinguiendo entre lo que es la recepción, ligada al proceso de matriculación, y una verdadera acogida, que implica actitudes hospitalarias y refuerza la implicación y fomento de la participación en las dinámicas educativas.

En relación al entorno, los planes de acogida municipales resultan claves para establecer procesos inclusivos. Igualmente, los sistemas de soporte son valorados positivamente por los docentes, pero también calificados como no suficientes. Resulta conveniente, al respecto, lograr una mayor coordinación entre los agentes implicados; por ejemplo, se hace necesario que los planes de entorno se elaboren a partir de la implicación de las familias y de todos los profesionales del centro educativo y no únicamente de los docentes. 
5. ANEXO

\begin{tabular}{|c|c|c|c|c|c|c|c|c|}
\hline \multicolumn{9}{|l|}{ MATRIZ DE INFORMACIÓN } \\
\hline ASPECTOS DE ANÁLISIS & \multicolumn{8}{|c|}{$\begin{array}{l}\text { FUENTES DE } \\
\text { INFORMACIÓN }\end{array}$} \\
\hline Instrumentos & 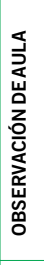 & 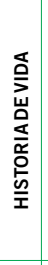 & 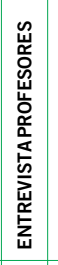 & 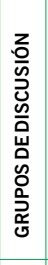 & 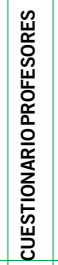 & 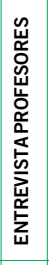 & 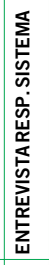 & 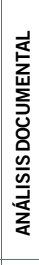 \\
\hline Protagonistas/contexto & 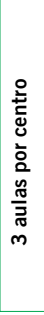 & 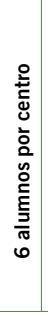 & 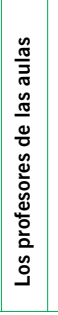 & 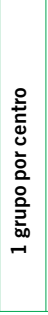 & 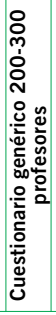 & 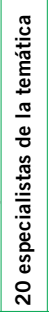 & 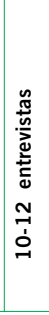 & 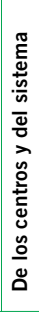 \\
\hline \multicolumn{9}{|l|}{ CONTEXTO ENSEÑANZA APRENDIZAJE } \\
\hline $\begin{array}{l}\text { Los objetivos se consideran comprometidos con los } \\
\text { estudiantes inmigrantes (El). }\end{array}$ & & & $\mathrm{X}$ & $\mathrm{X}$ & & & & $\mathrm{x}$ \\
\hline $\begin{array}{l}\text { Existen contenidos específicos para dar a conocer la } \\
\text { cultura y las características de los El. }\end{array}$ & & & $\mathrm{X}$ & $\mathrm{X}$ & & & & $\mathrm{x}$ \\
\hline $\begin{array}{l}\text { Existen actividades específicas para El con problemas } \\
\text { de aprendizaje. }\end{array}$ & $\mathrm{X}$ & & $x$ & $\mathrm{X}$ & & & & $\mathrm{X}$ \\
\hline $\begin{array}{l}\text { Existen actividades de tutoría individuales o colectivas } \\
\text { dirigidas a ofrecer soporte a los El. }\end{array}$ & $\mathrm{X}$ & & $\mathrm{X}$ & $\mathrm{X}$ & & & & $\mathrm{x}$ \\
\hline $\begin{array}{l}\text { La metodología promueve la interacción entre todos los } \\
\text { estudiantes. }\end{array}$ & $\mathrm{X}$ & $\mathrm{X}$ & $x$ & $\mathrm{X}$ & & & & $x$ \\
\hline \multicolumn{9}{|l|}{$\begin{array}{l}\text { La interacción del profesorado con los El en relación al } \\
\text { resto: }\end{array}$} \\
\hline Promueve/estimula su participación. & $\mathrm{X}$ & $\mathrm{x}$ & $x$ & $x$ & & & & \\
\hline Parte de aprendizajes previos de los estudiantes. & $\mathrm{X}$ & $\mathrm{X}$ & $x$ & $x$ & & & & \\
\hline $\begin{array}{l}\text { Considera y utiliza rasgos de su cultura en } \\
\text { explicaciones, ejemplos, etc. }\end{array}$ & $\mathrm{X}$ & $\mathrm{x}$ & $x$ & $x$ & & & & \\
\hline $\begin{array}{l}\text { Tiene en cuenta las singularidades de su contexto } \\
\text { familiar y social. }\end{array}$ & $\mathrm{X}$ & $\mathrm{X}$ & $\mathrm{X}$ & $\mathrm{X}$ & & & & \\
\hline $\begin{array}{l}\text { Opiniones sobre los tipos, características, } \\
\text { consecuencias y efectos de la interacción profesores- } \\
\text { estudiantes. }\end{array}$ & & & $\mathrm{X}$ & $X$ & & & & \\
\hline $\begin{array}{l}\text { Grado de discriminación implícita en los materiales } \\
\text { respecto a las culturas existentes en el aula. }\end{array}$ & $\mathrm{X}$ & $\mathrm{X}$ & $\mathrm{X}$ & $\mathrm{X}$ & & & & $x$ \\
\hline Existencia de materiales específicos. & $x$ & $x$ & $x$ & $\mathrm{x}$ & & & & $x$ \\
\hline $\begin{array}{l}\text { El sistema de evaluación tiene en cuenta posibles } \\
\text { déficits iniciales de los El. }\end{array}$ & & $x$ & $x$ & $\mathrm{X}$ & & & & $\mathrm{X}$ \\
\hline $\begin{array}{l}\text { Opiniones sobre el grado de consideración de los El en } \\
\text { el desarrollo de los procesos de enseñanza-aprendizaje } \\
\text { (aspectos comentados anteriormente). }\end{array}$ & & & & & $x$ & $x$ & $x$ & \\
\hline
\end{tabular}




\section{CONTEXTO AGENTES}

El estudiante inmigrante respecto al que no lo es tiene:

Sentimientos de estar discriminado.

Expectativas menores sobre el éxito en los estudios.

El estudiante no inmigrante respecto al inmigrante:

Asume la presencia de otras culturas y personas diferentes.

Se relaciona habitualmente con los El dentro de la escuela.

Se relaciona habitualmente con los El fuera de la escuela.

Su lenguaje es respetuoso.

Muestra actitudes de superioridad.

Promueve actividades de integración.

Las opiniones y actitudes del profesorado del aula:

Denotan estereotipos discriminatorios.

Refuerzan situaciones de predominio de unas culturas sobre otras.

Promueven diferencias entre los estudiantes.

Marginan a determinadas minorías.

Promueven actividades de integración.

Opiniones del profesorado sobre el grado de integración

de los El en el aula (aspectos anteriores).

\section{CONTEXTO AULA}

Decoración: refleja la diversidad, no discrimina, no reproduce estereotipos, etc.

Distribución de los El: concentración, dispersión, localización, etc.

Normas explícitas e implícitas: referencias a los El, a actuaciones a realizar, compensación de desigualdades, etc.

\section{Comportamientos:}

Grado de integración y de interrelación de los El en las actividades espontáneas.

Grado de integración y de interrelación de los El en las actividades programadas.

Actividades, referencias específicas respecto a los El

Diferencias de comportamiento de los profesores respecto a los El.

Diferencias de comportamiento en el trato para con los El dependiendo de las materias de aprendizaje.

Ambiente: estimulante, no coercitivo, respetuoso con los El, impulsor de la diversidad, etc.

Problemáticas existentes a nivel de aula y opciones adoptadas: aspectos anteriores $u$ otros vinculados.

Retos a futuro y medidas vinculadas: aspectos anteriores $\mathrm{u}$ otros relacionados.

\begin{tabular}{l|l|l|l|l|l|l|l|l} 
& $x$ & $x$ & $x$ & $x$ & & & & \\
\hline & $x$ & $x$ & $x$ & $x$ & & & & \\
\hline los & $x$ & $x$ & $x$ & $x$ & & & & $x$ \\
\hline & $x$ & $x$ & $x$ & $x$ & & & & $x$ \\
\hline & $x$ & $x$ & $x$ & $x$ & & & & \\
\hline & $x$ & $x$ & $x$ & $x$ & & & & \\
\hline & $x$ & $x$ & $x$ & $x$ & & & & \\
\hline & & & $x$ & $x$ & $x$ & $x$ & $x$ & $x$ \\
\hline
\end{tabular}




\section{CONTEXTO CENTRO}

Decoración: refleja la diversidad, no discrimina, no reproduce estereotipos, etc.

Distribución de los El: criterios de centro, situación del aula de acogida, etc.

Políticas explícitas e implícitas sobre El: referencias específicas en los planteamientos institucionales -Proyecto educativo de centro (PEC), proyecto curricular de centro (PCC), PA- actividades de centros para mejorar su integración.

Liderazgo: posición proactiva de los responsables de centros y equipos de profesores, influencia y control sobre sus actuaciones.

Profesorado: nivel de preparación y formación para tratar a los El, grado de trabajo en equipo, coordinación e innovación respecto de medidas para integrar alumnos inmigrantes.

Relaciones con la comunidad próxima: actividades específicas para familias de El, actividades de integración.

\begin{tabular}{|l|l|l|l|l|l|l|l}
$\mathrm{x}$ & $\mathrm{x}$ & $\mathrm{x}$ & $\mathrm{x}$ & & & & \\
$\mathrm{x}$ & $\mathrm{x}$ & $\mathrm{x}$ & $\mathrm{x}$ & & & & \\
\end{tabular}

lar

$x \times x \times$

Control de calidad: mecanismos de seguimiento, evaluación y rectificación sobre el tratamiento a los inmigrantes, resultados, efectividad.

Problemáticas existentes y opciones adoptadas (aspectos anteriores u otros vinculados).

Retos a futuro y medidas vinculadas: aspectos anteriores $u$ otros relacionados.

$x \quad x \quad x$

$x \quad x \quad x$

$x \quad x \quad x$

$X$

$x \quad x \quad x \quad x \quad x \quad x \quad x$

\section{CONTEXTO SISTEMA}

Filosofía del sistema: concentración-dispersión, normalización-atención individualizada, asimilacióninclusión.

Normativa vinculada a los El: referencias, requerimientos, sistemas de control.

Sistemas de soporte: tipología, características, funciones, requerimientos, sistemas de control.

Control de calidad: mecanismos de seguimiento, evaluación y rectificación sobre el tratamiento a El, resultados, efectividad.

Problemáticas existentes y opciones adoptadas: aspectos anteriores $u$ otros vinculados.

Retos a futuro y medidas vinculadas: aspectos anteriores $u$ otros relacionados). 


\section{BIBLIOGRAFÍA}

BESALÚ, Xavier (2004): «La formación inicial en interculturalidad», en J. A. JORDÁN y OTROS, La formación del profesorado en educación intercultural. Madrid: Los Libros de la Catarata.

-y CLIMENT, Teresa (2004): Construint identitats. Espais i processos de socialització dels joves d'origen immigrat. Barcelona: Mediterrànea.

BONAL, Xavier y OTROS (coords.) (2004): Política educativa i igualtat d'oportunitats. Prioritats i propostes. Barcelona: Mediterrànea.

CARBONELL, Francesc (coord.) (2000): Educació i immigració. Els reptes educatius de la diversitat cultural i l'exclusió social. Barcelona: Mediterrània.

CARBONELL, Jaume, SIMÓ, Núria y TORT, Antoni (2005): Magrebies en las aulas. Municipio, escuela e inmigración: un caso a debate. Vic: Eumo-Octaedro.

COMAS, Marta (2003): També catalans. Fills i filles de famílies immigrades a Catalunya. Barcelona: Fundació Jaume Bofill.

CONTEH, Jean (2003): Succeeding in Diversity. Culture, Language and Learning in Primary Classrooms. Stoke-on Trent: Trentham Books.

EURYDICE (2005): La integración escolar del alumnado inmigrante en Europa. Bruselas: Dirección General de Educación y Cultura, Comisión Europea. Disponible en: $<$ www.eurydice.org/ressources/eurydice/pdf/0_integral/045ES.pdf>.

FULLANA, Judit, BESALÚ, Xavier y VILÀ, Montserrat (2003): Alumnes d'origen africà a I'escola. Girona: C. Cardona Gamio (CCG).

FUSTER, Claudi (2004): La volta a l'escola en vuitanta mons. Diari d'un mestre de la diversitat. Barcelona: Associació de Mestres Rosa Sensat.

GARCIA, Eugene (2002): Student Cultural Diversity. Understanding and Meeting the Challenge. Boston: Houghton Mifflin.

GARRETA, Jordi (2003): El espejismo intercultural. La escuela de Cataluña ante la diversidad cultural. Madrid: Centro de Investigación y Documentación Educativa (CIDE).

GIMÉNEZ, Carlos y MALGESINI, Graciela (2002): Guía de conceptos sobre migraciones, racismo e interculturalidad. Madrid: Los Libros de la Catarata.

JORDÁN, José Antonio y OTROS (2004): La formación del profesorado en educación intercultural. Madrid: Los Libros de la Catarata.

LORENZO, Manuel y OTROS (coords.) (1999): Organización y dirección de instituciones educativas en contextos multiculturales. Una mirada a los países del Magreb desde Andalucía. Granada: Grupo Editorial Universitario.

MONTón, María José (2003): L'acolliment de l'alumnat d'incorporació tardana. Suggeriments, propostes i experiències. Barcelona: Graó.

PUJOLÀS, Pere (2004): Aprender juntos alumnos diferentes. Barcelona: Eumo-Octaedro. 
SIPÁN, Antonio (coord.) (2001): Educar para la diversidad en el siglo XXI. Zaragoza: Mira.

SUÁREZ-OROZCO, Carola y SUÁREZ-OROZCO, Marcelo (2003): La infancia de la inmigración. Madrid: Morata.

TORRABADELLA, Laura y TEJERO, Elisabet (2005): Pioneres i pioners. Trajectòries biogràfiques de filles $i$ fills de famílies immigrades a Catalunya. Barcelona: Mediterrània.

UNAMUNO, Virginia (2003): Lengua, escuela y diversidad sociocultural. Hacia una educación lingüística crítica. Barcelona: Graó.

VV. AA. (2000): Atención a la diversidad. Claves para la innovación educativa. Barcelona: Graó.

VV. AA. (2000): Com ens ho fem? Propostes per educar en la diversitat. Barcelona: Graó.

VV. AA. (2005): Estrategias organizativas de aula. Propuestas para atender la diversidad. Barcelona: Graó. 